\title{
Pilot Study of Younger and Older HIV-Infected Adults Using Traditional and Novel Functional Assessments
}

\author{
Uriel Sandkovsky ${ }^{1}$, Kevin R. Robertson ${ }^{2}$, Jane L. Meza ${ }^{3}$, Robin R. High ${ }^{3}$, Stephen J. \\ Bonasera $^{4}$, Christopher M. Fisher ${ }^{7}$, Anthony J. Marsh ${ }^{5}$, Margaret K. Sheehy ${ }^{5}$, Howard S. \\ Fox $^{6}$, and Susan Swindells ${ }^{1}$ \\ ${ }^{1}$ Department of Internal Medicine and Division of Infectious Diseases, University of Nebraska \\ Medical Center, Omaha, Nebraska \\ ${ }^{2}$ AIDS Neurological Center and Department of Neurology and Neuropsychology, School of \\ Medicine, University of North Carolina at Chapel Hill, Chapel Hill, North Carolina \\ ${ }^{3}$ Department of Biostatistics and Center for Collaboration on Research, Design and Analysis, \\ College of Public Health, University of Nebraska Medical Center, Omaha, Nebraska \\ ${ }^{4}$ Department of Internal Medicine and Division of Geriatrics, University of Nebraska Medical \\ Center, Omaha, Nebraska \\ ${ }^{5}$ Department of Health Promotion, Social \& Behavioral Health, College of Public Health, \\ University of Nebraska Medical Center, Omaha, Nebraska \\ ${ }^{6}$ College of Medicine, University of Nebraska Medical Center, Omaha, Nebraska \\ ${ }^{7}$ University of Nebraska Medical Center, Omaha, Nebraska
}

\section{Abstract}

Objectives-Emerging data suggest that HIV disease and its treatment affect the aging process. Accurate and reliable measures of functional status are needed to investigate this further.

Design-A pilot study in groups of younger and older HIV-infected adults using objective measures of function.

Methods-Evaluations included neuropsychological testing, grip strength, balance assessed by the Wii Balance Board, and actigraphy. Surveys were used for depression, fatigue, loneliness, selfreported activity level, and sexual function. Two-sample $t$ test or Wilcoxon rank sum tests were used for continuous variables and exact chi-square tests were used for comparison between groups.

Results-Twenty-one participants were 20 to 40 years old (younger; mean age, 31.5), and 20 were more than 50 years old (older; mean age, 56.5). There was no difference between groups for depression, fatigue, or loneliness. Overall, there was a trend to lower scores in the older age group for neuropsychological $z$ score $(P=.11)$ and for verbal learning $(P=.09)$. Functioning in the

(C) 2013 Thomas Land Publishers, Inc. www.hivclinicaltrials.com

Address for correspondence: Uriel Sandkovsky, MD, FACP, Assistant Professor of Medicine, Division of Infectious Diseases and HIV Clinic, University of Nebraska Medical Center, 988106 Nebraska Medical Center, Omaha, NE 68198-8106; phone: 402-559-8664; fax: 402-553-5527; usandkovsky@unmc.edu.

Previous publication: These data were presented in part at the Fourth International Meeting on HIV Infection and the Central Nervous System, Monte Porzio Catone (Rome), July 15-16, 2011, Italy, and the Second International Workshop on HIV \& Aging, October 27-28, 2011, Baltimore, MD.

Additional contributions: We thank Frances Van Meter and Jennifer O'Neill, study coordinators; Sebrina Taylor, laboratory technician; Deanna Hansen, administrative support; and all the participants. 
memory domain was significantly lower in older subjects $(P=.007)$. There was no difference in executive function, speed of processing, memory, motor skills, or total activity. Gender differences in sexual function were observed. Four older and 3 younger participants met the definition of frailty. Total activity by actigraphy did not correlate well with self-reported activity.

Conclusions-Objective tests were well accepted and feasible to perform, although not all are suitable for widespread clinical or research use. Objective measurements of activity did not correlate well with patient self-report, which has implications for future studies in this area.

\section{Keywords}

frailty; HIV; HIV and aging; HIV-associated neurocognitive disorders

By 2015, it is estimated that half the people living with HIV infection in the United States will be 50 years old or older. ${ }^{1}$ Emerging data suggest that chronically infected patients may be aging prematurely and experience an increased incidence of age-related conditions, including neurocognitive impairment and frailty. ${ }^{2-5}$ Even with effective antiretroviral treatment, older HIV-infected patients appear to be at risk for increased morbidity and mortality when compared to uninfected people with the same demographic characteristics. ${ }^{4,6,7}$ Debate continues about the relative contributions to the development of non-HIV-related conditions from the underlying HIV disease, from complications of longterm antiretroviral therapy, or from a cohort effect (ie, accounting for the underlying population age structure). ${ }^{1,8}$

There is growing evidence of ongoing chronic inflammation and immunosenscence, even in successfully treated HIV-infected patients, which likely contributes to the risk of comorbidities. ${ }^{9}$ Biomarkers such as IL-6, hsCRP, and D-dimer are elevated among HIVinfected patients compared to demographically matched uninfected control subjects, and they remain elevated even with successful antiretroviral therapy.${ }^{10}$ Comorbidities, including coronary heart disease, bone loss, liver cirrhosis, renal disease, ${ }^{1}$ and HIV-associated neurocognitive disorders, appear to persist despite effective treatment. ${ }^{5,11}$ Also of concern is the earlier occurrence of a frailty-related phenotype: a state of decreased physiologic reserve that increases the risk of morbidity and mortality. ${ }^{12-16}$ Although there is currently no accepted specific definition of "frailty" in HIV-infected patients, the frailty phenotype in the aged population is defined as the presence of at least 3 of the following characteristics: exhaustion, slowed walking speed, low activity level, weakness, and weight loss. ${ }^{13}$ Using this definition, in a cohort of HIV-infected men, the frailty phenotype before initiation of antiretroviral therapy predicted increased morbidity and mortality, independent of other risk factors. ${ }^{17}$ Although clinically useful, the frailty phenotype definition lacks rigorous metrics as patient self-report was used for assessments of activity, slowness, and exhaustion. 3,18,19

Ongoing and future investigations in this area must consider well-validated measures or clinical endpoints. The psychosocial and behavioral issues that often complicate the lives of people living with HIV infection also complicate the design of studies involving functional outcomes. We designed a pilot study incorporating innovative and traditional measures of cognitive and motor function, overall activity by actigraphy and self-report, emotional wellbeing, sexual function, inflammatory biomarkers, and frailty. We used objective measures whenever possible, and the primary objective of our study was to evaluate the feasibility of this approach. Secondary aims were to determine the optimal test battery to measure the areas of interest, to determine participant acceptance of the measures involved, and to collect preliminary data to inform future studies. 


\section{METHODS}

A cross-sectional, pilot study was conducted at the University of Nebraska Medical Center. Entry criteria were a diagnosis of HIV disease and, if taking antiretroviral therapy, on stable therapy for the past 12 weeks and not anticipated to require a change in therapy or, if not taking antiretroviral therapy, not anticipated to initiate therapy in the next 6 weeks. Participants were between 20 and 40 years of age or older than 50 years of age in order to clearly differentiate the age groups. Participants were required to be able to provide written informed consent and to complete the questionnaires in English, as not all the neuropsychological screens and questionnaires have been validated in other languages. Exclusion criteria were recent intercurrent acute infection, active psychiatric illness, active neurological disease, current delirium or intoxication, active drug or alcohol abuse, or pregnancy. Potential participants were approached with equal probability during scheduled clinic visits to the HIV clinic, and eligible participants were enrolled consecutively. NonEnglish-speaking patients were not approached. Only 1 person declined participation.

Participants' medical records were accessed to obtain demographic and medical history data, including information about any history of alcohol/substance abuse (which was also corroborated at the time of the participants' visit), educational and income levels, medication history for the last 90 days, and complete history for antiretroviral therapy. CD4+ T-cell count and HIV viral load was obtained from the medical records when available within 3 months or less of study entry, otherwise both were measured during study visits. Hepatitis $\mathrm{C}$ status was abstracted from the medical record, if available; otherwise, serological testing was done. Urine pregnancy testing was performed, if indicated.

All participants underwent history and physical examination including height and weight, calculated body mass index (BMI), and waist-to-hip ratio. Grip strength was measured using a bulb dynamometer; participants had 3 attempts with each hand and the average result was recorded. Balance was estimated using a Nintendo Wii Balance Board. ${ }^{20,21}$ Under supervision, participants were asked to complete balance exercises of increasing difficulty while standing on both feet at a fixed distance from a television monitor. Results were tabulated according to the number of successful levels completed.

Participants also underwent a validated neuropsychological (NP) battery to evaluate multiple domains most affected by HIV disease including executive function, motor skills, verbal learning, memory, and speed of processing. Specific instruments used were the Wide Range Achievement Test 4 (WRAT4) reading test, Timed Gait test, Hopkins Verbal Learning Test-Revised (HVLT-R), Trailmaking Test A and B, Grooved Pegboard Test, the Wechsler Adult Intelligence Scale (WAIS-3) Digit Symbol subtest, the Paced Auditory Serial Addition Test, Verbal or Letter Fluency, and the Stroop interference task. Normative standards were used that were corrected for age, education, sex and ethnicity, as appropriate, and scores were corrected using available computer programs. Validated questionnaires administered were the Beck Depression Inventory, the Fatigue Severity Scale (FSS) to measure self-reported fatigue and its impact on daily life, the UCLA Loneliness Scale to assess subjective feelings of loneliness or social isolation, and the Profile of Mood States (POMS), which includes self-reported activity.

Physical activity for 2 weeks was also measured using a portable, accelerometer-based activity monitor (Actiwatch-2; Philips Respironics, Murrysville, PA). ${ }^{22}$ Worn on the wrist, the device is equipped with a 3 -dimensional accelerometer and the signal is sampled at 30 $\mathrm{Hz}$, bandpass-filtered, and integrated to calculate activity counts at a 1-minute bin width. Algorithms have been developed to infer sleep schedule variability, sleep quantity and quality statistics, and daytime activity patterns for multiple days in an ambulatory 
environment. ${ }^{23,24}$ Participants were asked to use event markers on the device to record bedtime and arising time.

Sexual function was assessed using the Female Sexual Function Index ${ }^{25}$ (FSFI; a validated 19-item questionnaire that measures perceived desire, arousal, lubrication, orgasm, satisfaction, and pain) or the Male Sexual Health Questionnaire ${ }^{26}$ (MSHQ; a validated 16item questionnaire that measures perceived erection, ejaculation, and satisfaction).

Frailty was calculated using the phenotypic definition criteria from Fried et al. ${ }^{13}$ This definition consists of 3 or more of the following: > 10 lbs unintentional weight loss, exhaustion, low grip strength, slowed gait speed, and low physical activity. Plasma samples were collected for measurement of potential biomarkers (Myriad Rules Based Medicine; HumanMAP v1.6, Austin, TX). This included an 88-biomarker multi-analyte profile. Cellular markers such as soluble CD14, CD38, and HLA-DR were not included in the panel.

Participants were invited to provide feedback after the evaluations were completed. The local institutional review board approved the study, and all participants gave written informed consent.

\section{Statistical Analysis}

The primary outcome of the study was the proportion of patients who completed the full battery of tests. We anticipated that some subjects would fail screening; therefore, we planned to obtain consent from 50 patients and enroll up to 25 in each cohort. Patient characteristics and outcomes were descriptively summarized using means, medians, standard deviations, and frequencies. Descriptive analyses were conducted on all variables that were also visually assessed for outliers and missing data. Comparisons between the 2 groups were made with several types of statistical techniques, depending on the type of data (discrete or continuous). For discrete response data, odds ratios, or exact chi-square tests proportions, counts were analyzed with either Poisson or negative binomial models. The methods applied to continuous data were 2-sample $t$ tests; these were also checked for the presence of outliers, in which case the Wilcoxon 2-sample test was used. Time to event data were evaluated with the Cox proportional hazard model. Pearson or Spearman correlations were made with paired continuous data. All statistical significance tests were 2-sided. Statistical analyses were generated with SAS/STAT software, Version 9.3 of the SAS System for Windows (Cary, NC). Raw NP scores were standardized to $z$ scores (NPZ) to adjust for age and education.

\section{RESULTS}

Forty-one participants were recruited: 21 between 20 and 40 years old and 20 older than 50 years of age. There were 29 men and 12 women. Demographics of the participants are summarized in Table 1. The mean age of the younger group was 31.5 years; there were 14 men and 7 women, and 15 were White, 4 Black, and 2 Hispanic. The mean age of the older group was 56.5 years; 15 were men and 5 women; and 13 were White, 5 Black, and 2 Hispanic. There was no difference between groups for sexual orientation, education, income, tobacco/ substance abuse, most recent CD4 count, or viral load. Older patients had longer duration of HIV disease (mean 14.2 vs 5.9 years; $P<.001$, by Cox proportional hazards), lower CD4 nadir (mean 161.0 vs 315.2 cells $/ \mathrm{mm}^{3} ; P=.004$, by Wilcoxon 2 -sided nonparametric testing), longer antiretroviral therapy exposure (mean 11.3 vs 3.8 years; $P<$. 001, Wilcoxon 2-sided nonparametric testing), more diagnoses (mean 2.2 vs $0.8 ; P=.02$ ), and more concomitant medications (mean 4.3 vs $1.1 ; P=.004$, negative binomial). Only 2 participants had positive hepatitis $\mathrm{C}$ serology. 
There was $100 \%$ compliance with the study procedures among all participants with the exception of the Wii Balance Board tests, which 2 older participants could not complete. In addition, 4 participants did not wear the Actiwatch every day for the prescribed period of 2 weeks. With the exception of actigraphy, the complete test battery took about 2 hours to complete. The assessments required the purchase of the necessary equipment, and staff needed training to complete the neuropsychological tests. These tests required interpretation from a neuropsychologist, who was also responsible for ensuring interrater reliability of the staff members administering them.

Results of the assessments of grip strength, balance, neuropsychological performance, depression, loneliness, fatigue, and self-reported activity from the POMS are shown in Table 2. Participants were classified as having low grip strength if their measurement was below 1 standard deviation (SD) from the mean. The mean for the whole study group $(\mathrm{N}=41)$ was 32.1 pounds/square inch (psi), median of 32 (SD 8.0; SEM 1.25; 95\% CI, 29.7-34.8). Using this definition, 2 younger (10\%) and 6 older (30\%) subjects had low grip strength (Fisher's exact test, $P=.13$ ). About half the participants were able to complete 4 levels on the Wii Balance Board (67\% of younger and $50 \%$ of older participants). There was no difference in completion of the levels between the groups.

Regarding neuropsychological test scores, functioning in the memory domain was significantly lower in older subjects $(P=.007)$. Overall, there was a trend to lower overall neuropsychological test scores (NPZ) in the older age group $(P=.11)$ and for verbal learning $(P=.09)$. There was no evidence of difference in executive function, speed of processing, motor skills, or total activity between the younger and older groups. Despite a wide range of overall scores, there was no difference between the younger and older groups on the Beck's Depression Inventory scores. Mean values were below 17 (15.5 for the younger and 8.6 for the older), the level that indicates the presence of depression. ${ }^{27}$ Similarly, there was no difference in mean scores for self-reported fatigue using the Fatigue Severity Scale (34.3 vs 27.2) in which a score of 36 or less suggests that respondents are not suffering from fatigue. ${ }^{28}$ Scores on the UCLA Loneliness Scale of 15 to 20 are considered a normal experience of loneliness, and mean scores for both study groups approximated this range, with no significant difference between the groups. ${ }^{29}$

Actigraphy was recorded as total number of counts per 24-hour period (noon to noon). Days with activity counts below 20,000 were considered spurious, and participants with less than 5 days of valid data were excluded $(n=4)$. There were no significant differences between the younger and older groups for mean or median activity counts (Wilcoxon 2-sided nonparametric testing, $P=.97$ ) (Table 2 ). In addition, the overall ranges were similar. We did not observe differences in overall daily activity counts associated with participant age, gender, depression score, Loneliness Scale, or Fatigue Severity Score (data not shown). There was a trend that did not reach statistical significance suggesting that a higher depression score was associated with lower subject daily activity counts. Of particular note, actigraphy counts did not correlate with subject self-reported activity measured by the FSS and POMS scales (Spearman correlation $=0.25 ; P=.14$ ) (Figure 1A and 1B).

For the questionnaires on sexual function, younger HIV-infected men had higher scores on the erection scale than older men (mean 14.0 vs $9.5 ; 2$-sample $t$ test, $P<.001$ ) but also more concerns about erectile performance (odds ratio [OR], 0.082, indicating higher level of concern vs lower for older participants; $P=.0095$ ) (Table 3). Erection scores for HIVinfected participants correlated negatively with antiretroviral therapy $(P=.005)$, number of diagnoses $(P=.002)$, and concomitant medications $(P=.008)$ (data not shown). No evidence of differences was observed in other domains (ejaculation, satisfaction, desire). There was no difference between HIV-infected and uninfected, historical controls in 
erection, ejaculation, or satisfaction (means 9.5 vs $8.3,27.5$ vs 16.9 , and 22.7 vs 24.2 , respectively). ${ }^{26}$ Younger HIV-infected women reported more sexual desire compared to older women (2-sample $t$ test, mean 3.86 vs $1.92 ; P=.006$ ). No evidence of differences was observed in other domains (arousal, lubrication, orgasm, satisfaction, or pain). HIV-infected women had lower overall scores than uninfected, historical controls (mean 12.7 vs 19.2). We did not observe significant correlations with sexual function and NPZ score, fatigue, or loneliness or activity scores for men or women in either group (data not shown). Table 3 summarizes the responses to questionnaires about sexual function.

On the battery of biomarkers, the only significant differences between groups were seen in levels of alpha-1-antitrypsin, alpha fetoprotein, interleukin-8, vascular endothelial growth factor, and von Willebrand factor, which were all significantly higher in the older group (Figure 2). There was a trend for significantly higher levels of C-reactive protein, but no difference was found between groups for the rest of the biomarkers including interleukin- 6 or tumor necrosis factor-a.

To estimate the frailty phenotype, we used a Fatigue Severity Scale score of more than 36 to determine exhaustion; Timed Gait test results to evaluate walking speed, with greater than 11 seconds classified as slow; and the POMS self-reported activity scores. For POMS, activity is self-reported on a 5-point scale; we classified respondents indicating 0 to 1 as having low activity level. Using these criteria, 4 older (20\%) and 3 younger (14.3\%) participants met the definition of frailty.

Overall, the battery of tests was well received by the participants, and most feedback was positive, particularly for the assessments on the Wii. For the neuropsychological testing specifically, participants thought there were "too many tests" and majority of participants thought the Paced Auditory Serial Addition Test (PASAT) was too difficult, even demoralizing, and some refused to complete it.

\section{DISCUSSION}

The battery of tests used was well accepted and feasible to perform. However the more complex objective measurements, such as neuropsychological performance and actigraphy, require both specialized equipment and personnel for interpretation of results, which limit their utility in widespread clinical applications or even in the research setting. Simpler tools to identify neurocognitive impairment are available, such as the HIV Dementia Scale or a subscale of the Medical Outcomes Study HIV Health Survey (MOS-HIV), but these lack sensitivity for mild impairment. ${ }^{30}$ Similarly, activity is often measured by self-report, but the actigraphy results in our study correlated poorly with participants' self-reported activity and suggest that participants may overestimate how active they are. Most participants in our study complied with the directions to wear the device for 14 days and to use event markers on the device to record waking and sleeping times, although 4 of 41 participants did not. We also asked participants to keep a diary of the meal times and sleep/wake times when wearing the Actiwatch, as we thought this would help with interpretation of results. This proved counterproductive in the end, as several participants had difficulty remembering to enter information in the diary. After forgetting for a day or so, they gave up wearing the watch before the 2-week period ended, so we did not get complete data. The information from completed diaries was not particularly useful, and we did not have any difficulty with data interpretation from just the device. While actigraphy is a powerful tool to quantify activity level, is reproducible, and does not rely on self-perceived activity, the devices are expensive (almost \$1,000 each), which limits their use. 
We used a Wii Balance Board to evaluate balance; this is a more inexpensive and convenient measure than traditional balance testing with a laboratory-grade force platform, which is the gold standard but which has to be performed in a physical therapy department or similar setting. Currently, there is no inexpensive and portable system that precisely quantifies the center of pressure, an important component of standing balance. The Wii Balance Board fits these criteria; it has been evaluated in comparison with force platforms and found to be a valid tool for assessing standing balance. ${ }^{20}$ Although the Wii is widely available, portable, and inexpensive, custom-written software is required to interface the output with other programs to analyze the data in more detail. We used only the standard output from the Wii program for this study. More investigation is needed to fully assess the utility of this device, although it is already in common use in rehabilitation programs for patients with balance deficits and may have a role in interventional studies of frailty in HIV infection in future. ${ }^{21}$

We did not find differences between groups in terms of interleukin- 6 or tumor necrosis factor-a levels, but there was a trend for significantly higher C-reactive protein and alpha-1 antitrypsin levels and significantly higher interleukin- 8 von Willebrand and alpha fetoprotein levels in the older group. These findings may reflect the process of aging in the older group. We did not measure D-dimer, soluble CD14, or markers of T-cell activation in this pilot study due to logistics, but it is a consideration for a future study.

The frailty phenotype was commonly observed in this pilot study, with $20 \%$ in the older group and $14.3 \%$ in the younger group meeting the prespecified definition. Although the sample size is small, prevalence of frailty in men was higher than the rate published from the Multicenter AIDS Cohort Study (MACS) (15\% in a cohort of 863 men). We also observed higher than expected prevalence of frailty in the younger group of participants, almost as high as the older cohort in the MACS. ${ }^{31}$

In summary, the battery of tests used in this pilot study was well accepted and feasible to perform, although not all elements are suitable for widespread clinical or research use. Future investigations will need to balance the competing demands of rigorous, objective measurements and ease of administration and cost and complexity of interpretation. However, objective measurements of activity did not correlate well with patient self-report in our study, which has implications for future studies in this area.

\section{Acknowledgments}

Financial support/disclaimers: This work was supported by funding from National Institutes of Health, National Institute of Mental Health 5P30MH062261-12. Dr. Uriel Sandkovsky reports receipt of research grants to the University of Nebraska Medical Center from GlaxoSmithKline, Pfizer, and Behring for clinical trials unrelated to this project and has served as a consultant to Merck \& Co. Dr. Susan Swindells reports receipt of research grants to the University of Nebraska Medical Center from GlaxoSmithKline and Pfizer for clinical trials unrelated to this project. Robin R. High, Margaret Sheehy, Drs. Kevin R. Robertson, Howard S. Fox, Anthony Marsh, Jane L. Meza, Christopher Fisher, and Stephen J. Bonasera have nothing to report.

\section{References}

1. High KP, Brennan-Ing M, Clifford DB, et al. HIV and aging: State of knowledge and areas of critical need for research. A report to the NIH Office of AIDS Research by the HIV and Aging Working Group. J Acquir Immune Defic Syndr. 2012; 60(Suppl 1):S1-18. [PubMed: 22688010]

2. Deeks SG. HIV infection, inflammation, immunosenescence, and aging. Annu Rev Med. 2011; 62:141-155. [PubMed: 21090961]

3. Desquilbet L, Jacobson LP, Fried LP, et al. HIV-1 infection is associated with an earlier occurrence of a phenotype related to frailty. J Gerontol A Biol Sci Med Sci. 2007; 62(11):1279-1286.

[PubMed: 18000149] 
4. Guaraldi G, Orlando G, Zona S, et al. Premature age-related comorbidities among HIV-infected persons compared with the general population. Clin Infect Dis. 2011; 53(11):1120-1126. [PubMed: 21998278]

5. Heaton RK, Clifford DB, Franklin DR Jr, et al. HIV-associated neurocognitive disorders persist in the era of potent antiretroviral therapy: CHARTER Study. Neurology. 2010; 75(23):2087-2096. [PubMed: 21135382]

6. Hasse B, Ledergerber B, Furrer H, et al. Morbidity and aging in HIV-infected persons: The Swiss HIV cohort study. Clin Infect Dis. 2011; 53(11):1130-1139. [PubMed: 21998280]

7. Losina E, Schackman BR, Sadownik SN, et al. Racial and sex disparities in life expectancy losses among HIV-infected persons in the United States: Impact of risk behavior, late initiation, and early discontinuation of antiretroviral therapy. Clin Infect Dis. 2009; 49(10):1570-1578. [PubMed: 19845472]

8. Shiels MS, Pfeiffer RM, Engels EA. Age at cancer diagno sis among persons with AIDS in the United States. Ann Intern Med. 2010; 153(7):452-460. [PubMed: 20921544]

9. Hunt, PSE.; Epling, L.; Teague, J.; Tan, QX.; Martin, J.; Lum, P.; Deeks, S. T cell senescence and proliferation defects persist in treated HIV-infected individuals maintaining viral suppression and are associated with poor CD4+ T cell recovery [Abstract 316]. 17th Conference on Retroviruses and Opportunistic Infections; 2010; San Francisco, CA.

10. Neuhaus J, Jacobs DR Jr, Baker JV, et al. Markers of inflammation, coagulation, and renal function are elevated in adults with HIV infection. J Infect Dis. 2010; 201(12):1788-1795. [PubMed: 20446848]

11. Robertson KR, Smurzynski M, Parsons TD, et al. The prevalence and incidence of neurocognitive impairment in the HAART era. AIDS. 2007; 21(14):1915-1921. [PubMed: 17721099]

12. Bandeen-Roche K, Xue QL, Ferrucci L, et al. Phenotype of frailty: Characterization in the women's health and aging studies. J Gerontol A Biol Sci Med Sci. 2006; 61(3):262-266. [PubMed: 16567375]

13. Fried LP, Tangen CM, Walston J, et al. Frailty in older adults: Evidence for a phenotype. J Gerontol A Biol Sci Med Sci. 2001; 56(3):M146-156. [PubMed: 11253156]

14. Newman AB, Gottdiener JS, McBurnie MA, et al. Associations of subclinical cardiovascular disease with frailty. J Gerontol A Biol Sci Med Sci. 2001; 56(3):M158-166. [PubMed: 11253157]

15. Oursler KK, Sorkin JD, Smith BA, Katzel LI. Reduced aerobic capacity and physical functioning in older HIV-infected men. AIDS Res Hum Retroviruses. 2006; 22(11):1113-1121. [PubMed: 17147498]

16. Wanke CA, Silva M, Knox TA, Forrester J, Speigelman D, Gorbach SL. Weight loss and wasting remain common complications in individuals infected with human immunodeficiency virus in the era of highly active antiretroviral therapy. Clin Infect Dis. 2000; 31(3):803-805. [PubMed: 11017833]

17. Desquilbet L, Jacobson LP, Fried LP, et al. A frailty-related phenotype before HAART initiation as an independent risk factor for AIDS or death after HAART among HIV-infected men. J Gerontol A Biol Sci Med Sci. 2011; 66(9):1030-1038. [PubMed: 21719610]

18. Gill TM, Gahbauer EA, Allore HG, Han L. Transitions between frailty states among communityliving older persons. Arch Intern Med. 2006; 166(4):418-423. [PubMed: 16505261]

19. Woods NF, LaCroix AZ, Gray SL, et al. Frailty: Emergence and consequences in women aged 65 and older in the Women's Health Initiative Observational Study. J Am Geriatr Soc. 2005; 53(8): 1321-1330. [PubMed: 16078957]

20. Clark RA, Bryant AL, Pua Y, McCrory P, Bennell K, Hunt M. Validity and reliability of the Nintendo Wii Balance Board for assessment of standing balance. Gait Posture. 2010; 31(3):307310. [PubMed: 20005112]

21. Deutsch JE, Borbely M, Filler J, Huhn K, Guarrera-Bowlby P. Use of a low-cost, commercially available gaming console (Wii) for rehabilitation of an adolescent with cerebral palsy. Phys Ther. 2008; 88(10):1196-1207. [PubMed: 18689607]

22. Actiwatch-2. Murrysville, PA: Philips Respironics; http://www.healthcare.philips.com/pwc_hc/ main/homehealth/sleep/actiwatch/pdf/ActigraphyBrochure.pdf 
23. de Souza L, Benedito-Silva AA, Pires ML, Poyares D, Tufik S, Calil HM. Further validation of actigraphy for sleep studies. Sleep. 2003; 26(1):81-85. [PubMed: 12627737]

24. Gironda RJ, Lloyd J, Clark ME, Walker RL. Preliminary evaluation of reliability and criterion validity of Actiwatch Score. J Rehabil Res Dev. 2007; 44(2):223-230. [PubMed: 17551874]

25. Rosen R, Brown C, Heiman J, et al. The Female Sexual Function Index (FSFI): A multidimensional self-report instrument for the assessment of female sexual function. J Sex Marital Ther. 2000; 26(2):191-208. [PubMed: 10782451]

26. Rosen RC, Catania J, Pollack L, Althof S, O'Leary M, Seftel AD. Male Sexual Health Questionnaire (MSHQ): Scale development and psychometric validation. Urology. 2004; 64(4): 777-782. [PubMed: 15491719]

27. Beck AT, Steer RA, Ball R, Ranieri W. Comparison of Beck Depression Inventories -IA and -II in psychiatric outpatients. J Pers Assess. 1996; 67(3):588-597. [PubMed: 8991972]

28. Krupp LB, LaRocca NG, Muir-Nash J, Steinberg AD. The Fatigue Severity Scale. Application to patients with multiple sclerosis and systemic lupus erythematosus. Arch Neurol. 1989; 46(10): 1121-1123. [PubMed: 2803071]

29. Russell DW. UCLA Loneliness Scale (Version 3): Reliability, validity, and factor structure. J Pers Assess. 1996; 66(1):20-40. [PubMed: 8576833]

30. The Mind Exchange Working Group. Assessment, Diagnosis, and treatment of HIV-associated neurocognitive disorder: A consensus report of the Mind Exchange Program. Clin Infect Dis. published online ahead of print January 3, 2013.

31. Margolick, J.; Jacobson, L.; Lopez, J., et al. Frailty and circulating concentrations of proinflammatory cytokines and chemokines in HIV-infected and -uninfected men in the Multicenter AIDS Cohort Study (MACS) [Abstract O_13]. 3rd International Workshop on HIV and Aging; November 5-6, 2012; Baltimore, MD. 

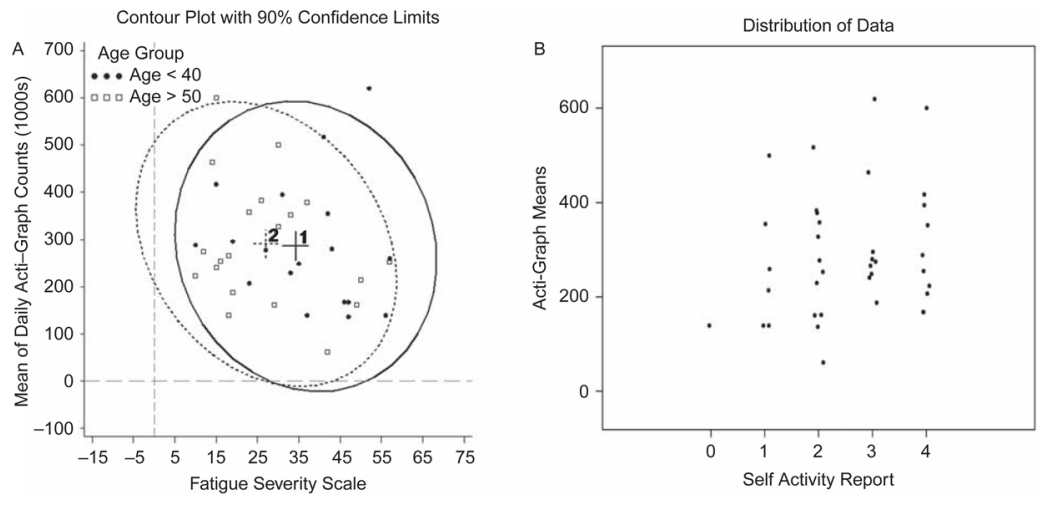

Figure 1.

(A) Contour plot with $90 \%$ CI comparing self-reported fatigue (using the Fatigue Severity Scale score) and actigraphy counts ( 1 unit $=1,000$ counts) demonstrating poor correlation. (B) Data distribution of self-reported activity for both younger and older participants (using the Profile of Mood States [PMS] activity score) and mean actigraphy counts ( 1 unit = 1,000 counts). The PMS score is reported as an ordinal activity level from 0 to 4 , with 0 being the least active and 4 the most active. There was no difference between actigraphy counts across the 4 self-reported activity levels. Similarly, no difference was seen between older and younger participants (data not shown). 

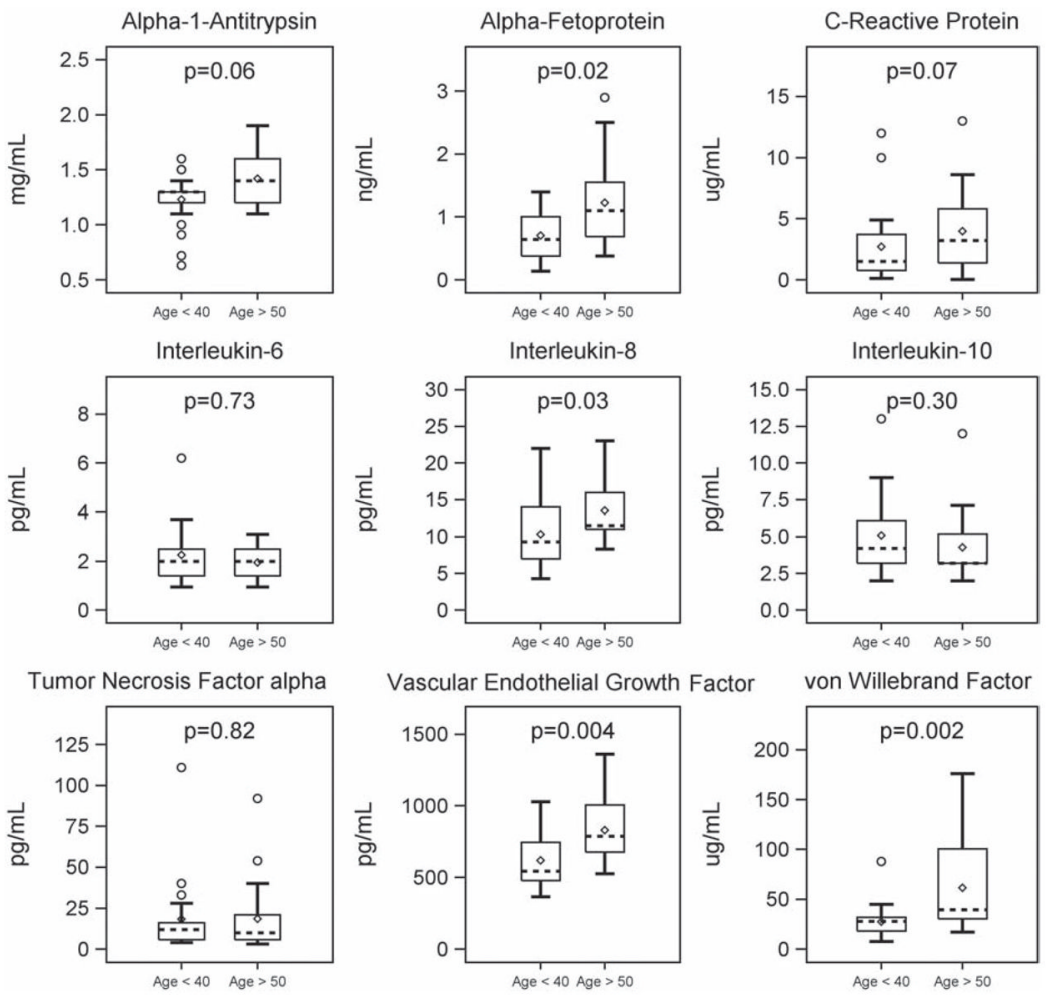

Figure 2.

Comparison of selected biomarkers level by older or younger group with $P$ values calculated using Wilcoxon rank sum test. 
Table 1

Participant characteristics

\begin{tabular}{|c|c|c|c|}
\hline & Younger $(<40$ years old $) n=21$ & Older $(>50$ years old $) \mathbf{n = 2 0}$ & $P$ value $^{a}$ \\
\hline Mean age, years (range) & $31.5(20-40)$ & $56.2(50-70)$ & \\
\hline \multicolumn{4}{|l|}{ Gender } \\
\hline Male & $14(67)$ & $15(75)$ & .73 \\
\hline Female & $7(33)$ & $5(25)$ & \\
\hline \multicolumn{4}{|l|}{ Race } \\
\hline White & $15(71)$ & $13(65)$ & .89 \\
\hline Black & $4(19)$ & $5(25)$ & \\
\hline Hispanic & $2(10)$ & $2(10)$ & \\
\hline \multicolumn{4}{|l|}{ Sexual orientation } \\
\hline MSM & $14(66)$ & $10(50)$ & \\
\hline Heterosexual & $7(33)$ & $6(30)$ & \\
\hline Bisexual & 0 & $4(20)$ & \\
\hline Mean years of education & 13.5 & 13.9 & $.92^{b}$ \\
\hline Mean income level $(1-4)^{c}$ & 2.2 & 2.3 & .97 \\
\hline Current tobacco use & $9(43)$ & $7(35)$ & .79 \\
\hline \multicolumn{4}{|l|}{ Substance abuse } \\
\hline Current & $2(10)$ & $3(15)$ & .18 \\
\hline Past & $11(52)$ & $5(25)$ & \\
\hline Never & $8(38)$ & $12(60)$ & \\
\hline Mean years since HIV diagnosis (range) & $5.9(0.0-12.9)$ & $14.2(1.6-24.0)$ & $<.001^{d}$ \\
\hline Mean CD4 nadir in cells $/ \mathrm{mm}^{3}$ (range) & $315.2(12-852)$ & $161.0(7-569)$ & $.0045^{b}$ \\
\hline Mean current CD4 (within last 3 months) & $706(135-1,167)$ & $657.8(185-1,496)$ & $.99^{b}$ \\
\hline Viral load $<50$ copies $/ \mathrm{mL}$ & $15(71)$ & $19(95)$ & .09 \\
\hline Mean length of time on ART in years (range) & $3.8(0-12)$ & $11.3(1.5-18)$ & $<.001$ \\
\hline Mean no. of diagnoses apart from HIV infection (range) & $0.8(0-3)$ & $2.2(0-6)$ & $.02^{b}$ \\
\hline Mean no. of concomitant medications excluding ART, (range) & $1.1(0-6)$ & $4.3(0-28)$ & $.004^{e}$ \\
\hline
\end{tabular}

Note: Values are given as $\mathrm{n}(\%)$, unless otherwise indicated. ART = antiretroviral therapy; MSM = men having sex with men.

$a_{P}=$ exact Poisson.

${ }^{b}$ Wilcoxon 2-sample nonparametric test.

${ }^{c}$ Income level: $1=$ living at or below the Federal Poverty Level (FPL); $2=200 \%$ of the FPL; $3=300 \%$ of the PFL; $4=400 \%$ of the FPL or more.

$d_{\text {Cox proportional hazards. }}$

$e^{e}$ Negative binomial. 
Table 2

Results of the assessments of grip strength, balance, neuropsychological performance, depression, loneliness, fatigue, self-reported activity, and counts measured by actigraphy

\begin{tabular}{|c|c|c|c|}
\hline & Younger $(<40$ years old $) n=21$ & Older $(>50$ years old $) n=20$ & $P$ value \\
\hline \multirow[t]{3}{*}{ Combined grip strength men (psi) } & Mean $=37.2$ & Mean $=33.6$ & .17 \\
\hline & Median $=38.0$ & Range $=32.7$ & \\
\hline & Range $=27.4-47.0$ & Range $=23.7-44.0$ & \\
\hline \multirow[t]{3}{*}{ Combined grip strength women (psi) } & Mean $=27.7$ & Mean $=19.7$ & .03 \\
\hline & Median $=27.8$ & Median $=21$ & \\
\hline & Range $=23.3-33$ & Range $=14.3-24$ & \\
\hline \multirow[t]{6}{*}{ Wii Balance Board } & Not completed $=0(0 \%)$ & Not completed $=2(10 \%)$ & NS \\
\hline & Level $1=0(0 \%)$ & Level $1=1(5 \%)$ & \\
\hline & Level $2=2(10 \%)$ & Level $2=2(10 \%)$ & \\
\hline & Level $3=5(24 \%)$ & Level $3=5(25 \%)$ & \\
\hline & Level $4=12(57 \%)$ & Level $4=9(45 \%)$ & \\
\hline & Level $5=2(10 \%)$ & Level $5=1(5 \%)$ & \\
\hline NPZ score & -.0 .29 & -0.73 & .11 \\
\hline Motor & -0.49 & -0.47 & .95 \\
\hline Verbal learning & -0.28 & -0.98 & .09 \\
\hline Memory domain & -0.2 & -1.25 & .007 \\
\hline Executive function & -0.16 & 0.03 & .49 \\
\hline Speed of processing & -0.024 & -0.15 & .62 \\
\hline Attention & -0.59 & -1.75 & .18 \\
\hline \multirow[t]{2}{*}{ Beck Depression Inventory score } & Mean $=15.5$ & Mean $=8.6$ & .17 \\
\hline & Range $=0-50$ & Range $=0-22$ & \\
\hline \multirow[t]{2}{*}{ UCLA Loneliness Scale } & Mean $=20.7$ & Mean $=17.8$ & .49 \\
\hline & Range $=10-35$ & Range $10-33$ & \\
\hline \multirow[t]{2}{*}{ Fatigue Severity Scale } & Mean $=34.3$ & Mean $=27.2$ & .14 \\
\hline & Range $=10-57$ & Range $=10-57$ & \\
\hline \multirow[t]{2}{*}{ POMS activity level } & Mean $=2.7$ & Mean $=2.5$ & \\
\hline & Range $=0-4$ & Range $=1-4$ & \\
\hline \multirow[t]{2}{*}{ Actigraphy: daily counts x 1,000} & Mean $=285.5$ & Mean $=290.0$ & .97 \\
\hline & Range $=136.3-619.7$ & Range $=61.3-600.3$ & \\
\hline
\end{tabular}

Note $: \mathrm{NPZ}=$ Neuropsychological $z$ score$;$ POMS $=$ Profile of Mood States $;$ psi $=$ pounds $/$ square inch. 


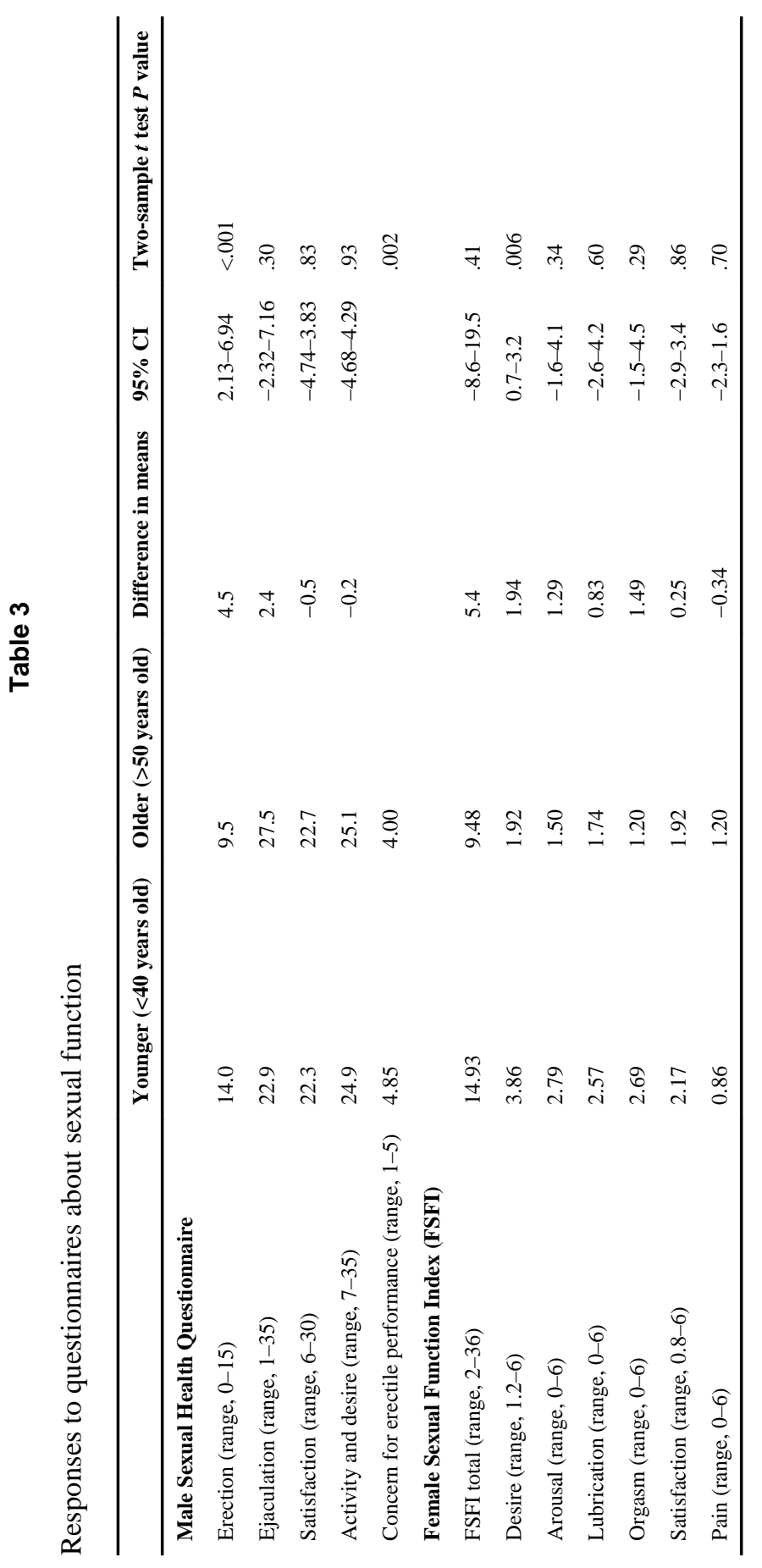

HIV Clin Trials. Author manuscript; available in PMC 2014 February 21. 